\title{
Гигантский эффект выпрямления терагерцового излучения в периодических графеновых плазмонных структурах
}

\author{
(C) Д.В. Фатеев ${ }^{1}$, К.В. Машинский ${ }^{1,2}$, Hua Qin ${ }^{3}$, Jiandong Sun ${ }^{3}$, B.В. Попов ${ }^{1,2,4}$ \\ ${ }^{1}$ Саратовский фрилиал Института радиотехники и электроники им. В.А. Котельникова Российской академии наук, \\ 410019 Саратов, Россия \\ ${ }^{2}$ Саратовский государственный университет им. Н.Г. Чернышевского, \\ 410012 Саратов, Россия \\ ${ }^{3}$ Key Laboratory of Nanodevices and Applications, \\ Suzhou Institute of Nano-tech and Nano-bionics (SINANO), Chinese Academy of Sciences, \\ 398 Ruoshui Road, 215123 Suzhou, P. R. China \\ ${ }^{4}$ Саратовский научный центр Российской академии наук, \\ 410028 Саратов, Россия \\ E-mail: FateevDV@yandex.ru
}

(Получена 27 апреля 2017 г. Принята к печати 12 мая 2017 г.)

\begin{abstract}
Рассмотрено выпрямление терагерцового излучения за счет плазмонных нелинейностей в периодической графеновой структуре с двойным решеточным затвором и асимметричной элементарной ячейкой. Исследовано влияние асимметрии элементарной ячейки на плазмонное выпрямление терагерцового излучения вблизи высших плазмонных резонансов для случаев электронной и дырочной проводимости на участках элементарной ячейки графена. Вычислена токовая чувствительность выпрямления терагерцового излучения за счет плазмонных эффектов дифференциального увлечения носителей заряда и электронно-дырочного храповика.
\end{abstract}

DOI: 10.21883/FTP.2017.11.45110.24

\section{1. Введение}

Плазменные волны (плазмоны) в двумерных (2D) электронных системах могут быть использованы для генерации, усиления, преобразования и детектирования терагерцового (ТГц) излучения, поскольку частоты плазменных резонансов находятся в ТГц частотном диапазоне для субмикрометровых размеров 2D резонаторов [1]. За счет своей классической природы, высокой скорости по сравнению со скоростью движения электрона в полупроводниковых структурах и нелинейных свойств плазмоны представляются перспективными для использования в ТГц диапазоне частот. Основное ограничение на распространение плазмонов в 2D системах накладывает быстрое рассеяние импульса носителей заряда при комнатной температуре, однако в современных материалах, таких как GaN/AlGaN [2], GaAs/InGaAs [3] и графен [4], подвижность свободных носителей заряда достигает величин [5], при которых наблюдаются плазмонные эффекты при комнатной температуре.

В направлении создания плазмонных детекторов ТГц излучения усилия сосредоточены как на использовании ТГц плазмонов в качестве концентраторов ТГц поля [6-8], так и на применении нелинейных свойств плазменных волн в 2D системах для выпрямления ТГц излучения $[9,10]$. В последнее время активно исследуется плазмонное детектирование ТГц излучения в 2D электронных системах с решеточным затвором [11]. Плазмонное детектирование в полупроводниковых гетероструктурах с 2D электронными системами связано c эффектами увлечения носителей заряда плазмонами и плазмонного храповика [12]. Рекордные значения чувствительности были достигнуты при использовании плазмонного детектирования ТГц излучения в транзисторах с высокой подвижностью электронов, имеющих двойной решеточный затвор [13].

Исследования плазмонных детекторов на основе графена главным образом посвящены структурам с одиночным затворным электродом, в которых эффективность возбуждения плазмонов достаточно низкая $[14,15]$. Решеточный затвор как один из наиболее эффективных способов возбуждения 2D плазмонов применялся в плазмонных графеновых структурах для выпрямления ТГц излучения в нерезонансном режиме [4]. Плазмонное выпрямление ТГц излучения в графене с решеточным затвором возникает в результате действия двух физических механизмов [10]: эффекта дифференциального увлечения носителей заряда полем плазмона и эффекта электронно-дырочного плазмонного храповика.

В данной работе рассмотрено выпрямление ТГц излучения в графеновой структуре с двойным решеточным затвором при резонансном возбуждении плазмонов вблизи высших плазмонных резонансов. Исследовано влияние геометрической асимметрии элементарной ячейки и асимметрии электрического поля на плазмонное выпрямление ТГц излучения вблизи высших плазмонных резонансов. Вычислена токовая чувствительность выпрямления ТГц излучения за счет плазмонных эффектов в графеновой структуре с двойным решеточным затвором.

\section{2. Теоретическая модель}

Рассмотрим структуру, состоящую из графена, расположенного на поверхности подложки $\mathrm{SiO}_{2}$ и отделен- 


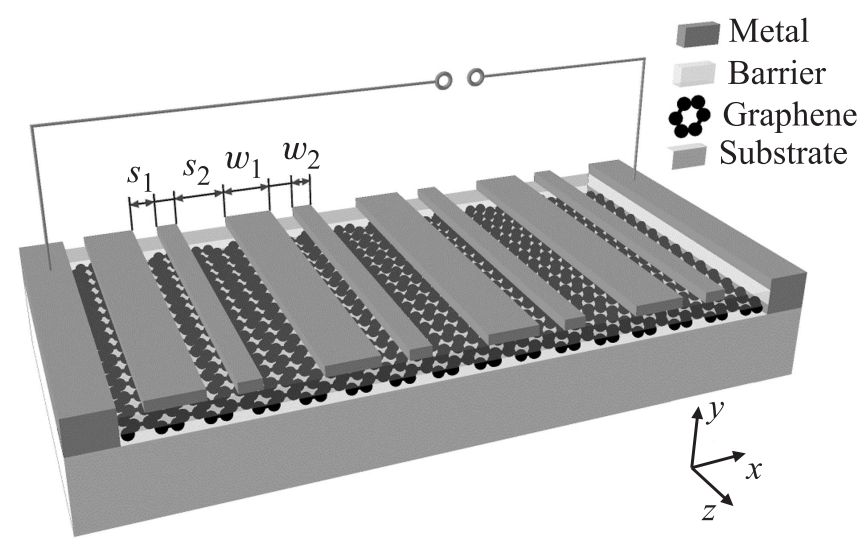

Рис. 1. Схематическое изображение графеновой структуры с двойным решеточным затвором.

ного от двойного металлического решеточного затвора барьерным слоем $\mathrm{Al}_{2} \mathrm{O}_{3}$ (см. рис. 1). Две подрешетки затвора расположены в одной плоскости и смещены друг относительно друга для создания асимметрии элементарной ячейки периодической структуры. Приложение постоянного напряжения между затвором и графеном позволяет контролировать равновесную концентрацию носителей заряда в подзатворных областях графена. От знака приложенного напряжения зависит тип носителей заряда в подзатворной области графена.

Нелинейные свойства движения носителей заряда в графене описываются в гидродинамическом приближении [16]. Гидродинамические уравнения решаются с использованием теории возмущений [17] путем разложения скорости носителей заряда, энергии Ферми и плотности тока по степеням амплитуды действующего электрического поля с сохранением линейных и квадратичных членов разложения. Используются приближения нулевой температуры и малой скорости носителей заряда. С использованием данного подхода получим выражение для вычисления плотности выпрямленного тока в графеновой структуре [10]:

$$
\begin{aligned}
j_{0}= & -\frac{\left|e^{3} v_{\mathrm{F}}^{2}\right|}{2 \hbar^{2} \pi \omega \gamma\left(\omega^{2}+\gamma^{2}\right)} \\
& \times\left\langle\operatorname{sgn}(e) \operatorname{Re}\left[\left(\omega-i \frac{\gamma}{2}\right) E_{x, \omega} \frac{\partial\left(E_{x, \omega}\right)^{*}}{\partial x}\right]\right\rangle,
\end{aligned}
$$

где $e-$ элементарный заряд ( $e>0$ для дырок и $e<0$ для электронов), $E_{x, \omega}-x$-компонента электрического поля, действующего на носители заряда в графене, $\omega-$ круговая частота поля, $\gamma-$ скорость релаксации импульса носителей заряда, угловые скобки означают пространственное усреднение по элементарной ячейке периодической графеновой структуры.

Будем считать, что на участке графена под затвором с металлическими полосками шириной $w_{2}$ имеется проводимость одного типа (например, дырочного), а в остальной части элементарной ячейки - проводи- мость другого типа (электронного). Такое пространственное распределение носителей в графене описывается распределением равновесной энергии Ферми $\varepsilon_{\mathrm{F}, w_{1}}^{(0)}=\varepsilon_{\mathrm{F}, s_{1}}^{(0)}=\varepsilon_{\mathrm{F}, s_{2}}^{(0)}=\varepsilon_{\mathrm{F} 1}$ и $\varepsilon_{\mathrm{F}, w_{2}}^{(0)}=\varepsilon_{\mathrm{F} 2}$. Для периодической структуры с указанным распределением носителей заряда выражение (1) может быть преобразовано к виду

$$
j_{0}=j_{0}^{d r}+j_{0}^{r t}
$$

где

$$
\begin{aligned}
j_{0}^{d r}= & \frac{|e|^{3} v_{\mathrm{F}}^{2}}{\hbar^{2} \pi \omega\left(\omega^{2}+\gamma^{2}\right)} \\
\times & \left.\operatorname{sgn}\left(\varepsilon_{\mathrm{F} 1}\right)\left[1-\frac{w_{2}}{l}\right]+\operatorname{sgn}\left(\varepsilon_{\mathrm{F} 2}\right) \frac{w_{2}}{l}\right\} \sum_{p} q_{p}\left|E_{x, \omega, p}\right|^{2}, \\
j_{0}^{r t}= & \frac{|e|^{3} v_{\mathrm{F}}^{2}}{\hbar^{2} \pi \omega\left(\omega^{2}+\gamma^{2}\right)} \frac{4}{\gamma l}\left[\operatorname{sgn}\left(\varepsilon_{\mathrm{F} 2}\right)-\operatorname{sgn}\left(\varepsilon_{\mathrm{F} 1}\right)\right] \\
& \times \operatorname{Re}\left\{\left(i \omega+\frac{\gamma}{2}\right) \sum_{p} \sum_{p^{\prime} \neq p} E_{x, \omega, p} E_{x, \omega, p^{\prime}}^{*} \frac{p^{\prime}}{p-p^{\prime}}\right. \\
& \left.\times \sin \left[\pi\left(p-p^{\prime}\right) \frac{w_{2}}{l}\right] \exp \left[i \pi\left(p-p^{\prime}\right) \frac{s_{1}-s_{2}}{l}\right]\right\},
\end{aligned}
$$

где использовано разложение электрического поля в пространственный ряд Фурье

$$
E_{x, \omega}(x)=\sum_{p} E_{x, \omega, p} \exp \left(i q_{p} x\right) \quad \text { и } \quad q_{p}=2 \pi p / l,
$$

$p, p^{\prime}$ - целые числа. Выпрямление ТГц излучения в периодической графеновой структуре может происходить за счет двух разных физических механизмов. Одним из физических механизмов является выпрямление ТГц излучения за счет эффекта дифференциального плазмонного увлечения носителей заряда в графене [10], при этом выпрямленный ток увлечения вычисляется с помощью выражения (3). Необходимым условием существования эффекта плазмонного увлечения является асимметрия пространственного распределения электрического поля, $\left|E_{x, \omega, p}\right| \neq\left|E_{x, \omega,-p}\right|$. Заметим, что выражение для вычисления плотности тока увлечения в графене с участками электронной и дырочной проводимости отличается от известного выражения для плотности тока увлечения в графене с одним типом носителей заряда [10] наличием множителя с усреднением функции знака равновесной энергии Ферми по длине элементарной ячейки графена (см. выражение (3)). Следовательно, для структур с равными размерами участков графена с электронной и дырочной проводимостью выпрямленный ток увлечения равен нулю. Вторым механизмом выпрямления ТГц излучения в графене выступает эффект плазмонного электронно-дырочного храповика [10] с плотностью выпрямленного тока, определяемой выражением (4). Выпрямление ТГц излучения за счет эффекта плазмонного электронно-дырочного храповика возможно только в 
графене с участками с разным типом проводимости в элементарной ячейке (см. выражение (4)). На эффективность выпрямления ТГц излучения за счет эффекта электронно-дырочного плазмонного храповика влияет как геометрическая асимметрия графеновой структуры, так и взаимодействие пространственных гармоник электрического поля разных порядков. Эффективное взаимодействие пространственных гармоник электрического поля разных порядков возможно при возбуждении в структуре гибридных плазмонных резонансов в результате взаимодействия плазмонных мод разных плазмонных резонаторов в элементарной ячейке графеновой структуры $[18,19]$. В пространственно-асимметричной структуре в дополнение к, „радиационным“ плазмонным модам с волновым вектором, кратным ширинам плазмонных резонаторов, $k_{p}=(2 n-1) \pi / w_{1,2}$, возбуждаются „нерадиационные“ плазмонные моды, волновой вектор которых определяется соотношением $k_{p}=2 \pi n / w_{1,2}$ [19], где $n-$ номер плазмонной моды.

Токовая чувствительность детектирования ТГц излучения на единицу площади графеновой структуры определяется выражением $R=j_{0} / P$, где $P$ - плотность мощности ТГц волны, падающей на графеновую структуру.

\section{3. Результаты и их обсуждение}

Плазмонное выпрямление ТГц излучения исследовано для графеновой структуры с двойным решеточным затвором (рис. 1) с параметрами $w_{1}=1$ мкм, $w_{2}=0.25$ мкм, $\gamma=0.2 \cdot 10^{-12} \mathrm{c}^{-1}$ для распределения энергии Ферми $\varepsilon_{\mathrm{F}, w_{1}}^{(0)}=\varepsilon_{\mathrm{F}, s_{1}}^{(0)}=\varepsilon_{\mathrm{F}, s_{2}}^{(0)}=-\varepsilon_{\mathrm{F}, w_{2}}^{(0)}=39$ мэВ. Рассмотрена геометрически асимметричная структура, в которой длины просветов $s_{1}$ и $s_{2}$ между электродами затвора изменяются, а суммарная ширина просветов остается постоянной $s_{1}+s_{2}=0.375$ мкм. Электрические поля плазмонов, возбуждаемые падающей ТГц волной $\left(E_{\text {ext }}\right)$, и коэффициенты поглощения для рассматриваемой графеновой структуры вычислены в рамках самосогласованного электродинамического подхода, основанного на методе интегрального уравнения [18]. Спектры коэффициентов поглощения и токовых чувствительностей детектирования рассчитаны для разных коэффициентов асимметрии структуры $K=1-s_{1} / s_{2}$, $0<K<1$ (рис. 2). На рис. 2, $b$ показан суммарный эффект выпрямления ТГц излучения за счет двух плазмонных механизмов, описываемых выражениями (3) и (4), однако вклад эффекта увлечения носителей заряда полем плазмона в суммарный выпрямленный ток на 2 порядка величины меньше вклада эффекта плазмонного электронно-дырочного храповика.

Эффект выпрямления ТГц излучения в плазмонном резонансе на частоте 1 ТГц, соответствующий резонансному возбуждению первой плазмонной моды в графене под широким затвором, рассмотрен в работе [10]. В первом плазмонном резонансе (на частоте 1 ТГц) и во втором резонансе (на частоте 2 ТГц) в данной

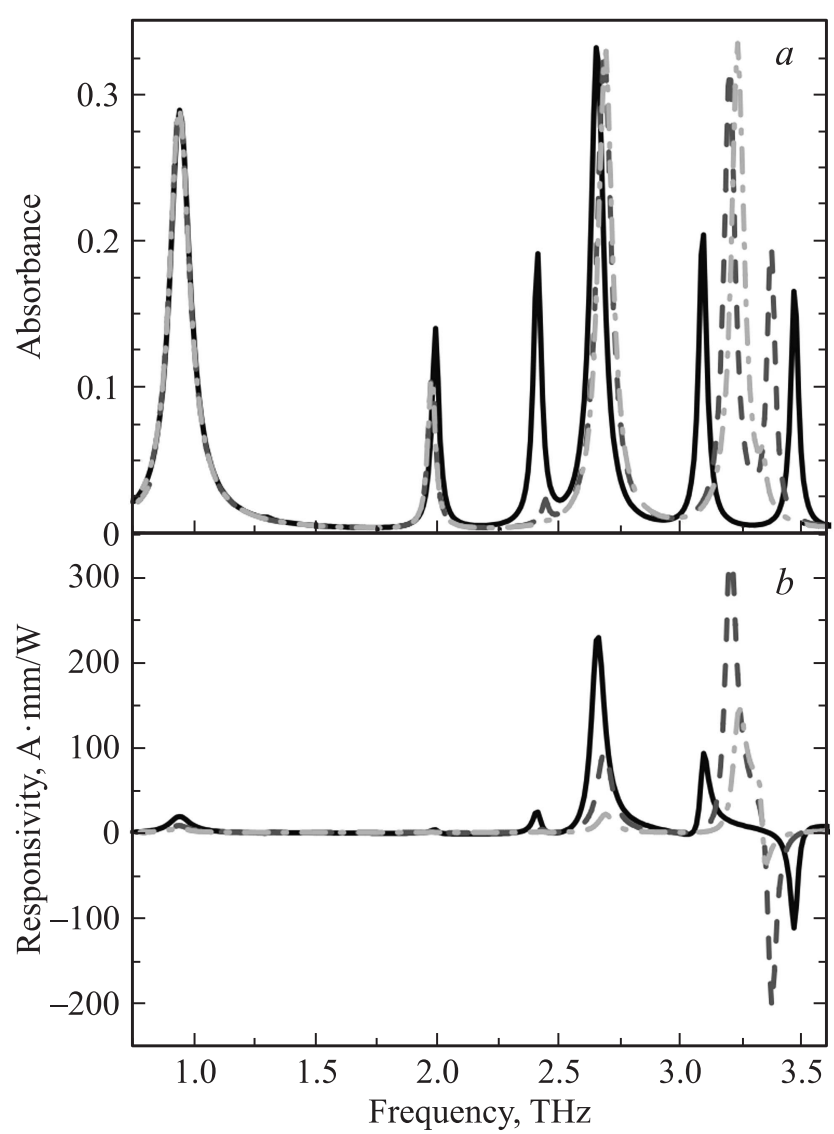

Рис. 2. Спектры коэффициента поглощения графеновой структуры $(a)$ и спектры токовой чувствительности при различных значениях коэффициента асимметрии $(b)$. Сплошные кривые $-K=0.1$, штриховые $-K=0.4$, штрихпунктирные $K=0.9$.

структуре возбуждаются подзатворные плазмонные моды под широким затвором, слабо взаимодействующие с другими плазмонными модами. В этом случае поля плазмонов обладают симметрией [19] и поэтому эффект плазмонного электронно-дырочного храповика определяется в основном пространственной геометрической асимметрией графеновой структуры, а именно продольным смещением области с дырочной проводимостью относительно центра широкого затворного электрода (который определяет профиль электрического поля в структуре).

Влияние асимметрии электрического поля в эффекте плазмонного электронно-дырочного храповика видно на примере третьего и четвертого резонансов на частотах 2.4 и 2.7 ТГц. Резонансы на этих частотах представляют собой две взаимодействующие плазмонные моды, а именно являются комбинацией второй „нерадиационной“ плазмонной моды под широким затворным электродом шириной $w_{1}$ и первой ,„радиационной“ плазмонной моды под узким затвором шириной $w_{2}$ (рис. 3 и 4). При увеличении геометрической асимметрии структуры влияние „нерадиационной“ плазмонной моды растет (рис. 3), что приводит к росту асимметрии электрическо- 


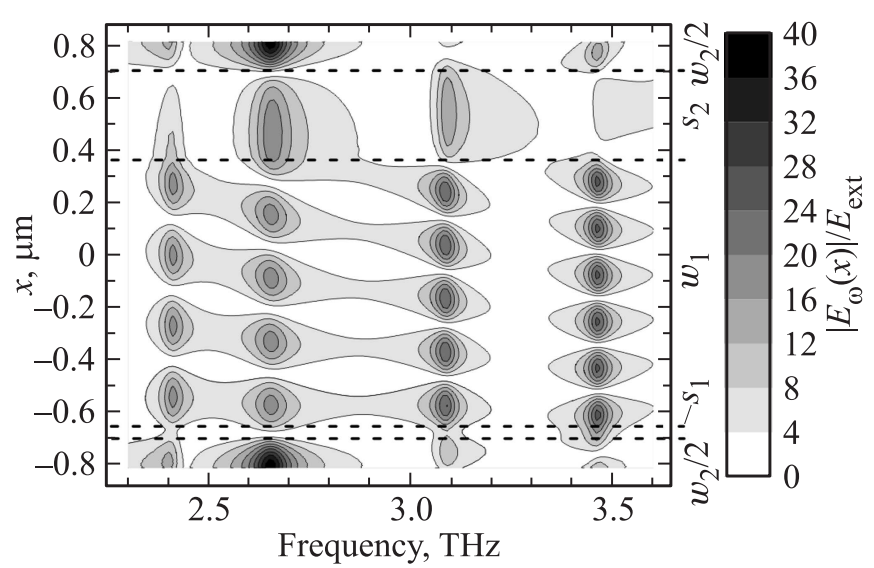

Рис. 3. Пространственное распределение электрического поля плазмонов $E_{\omega}$ в элементарной ячейке графеновой структуры при коэффициенте асимметрии $K=0.9$.

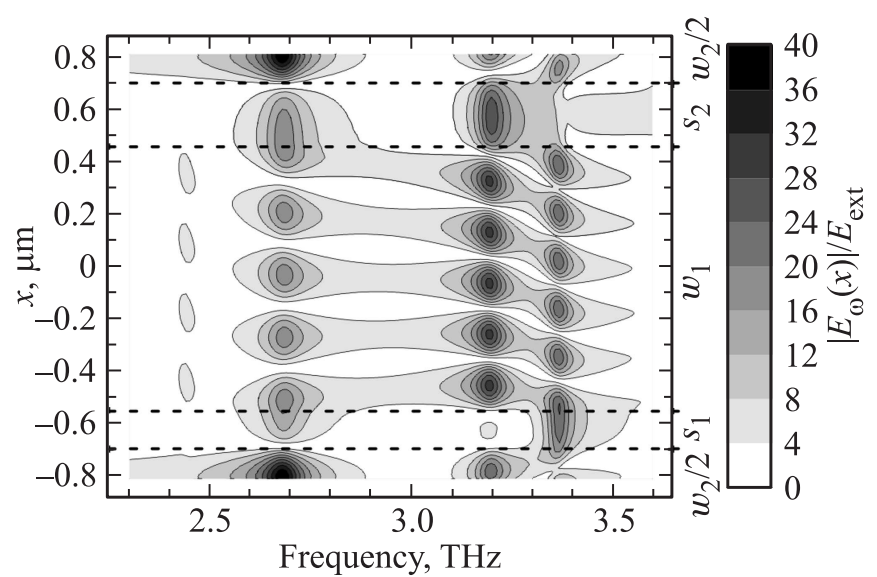

Рис. 4. Пространственное распределение электрического поля плазмонов $E_{\omega}$ в элементарной ячейке графеновой структуры при коэффициенте асимметрии $K=0.4$.

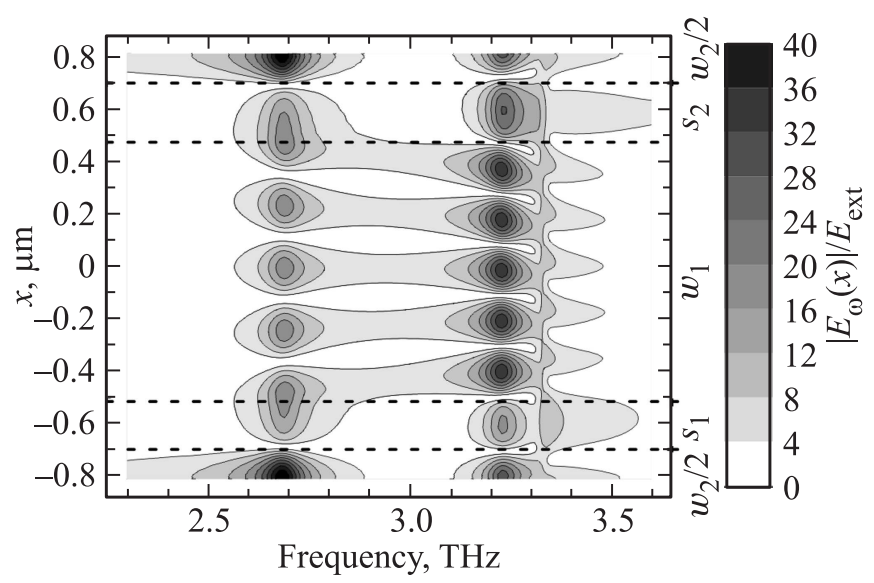

Рис. 5. Пространственное распределение электрического поля плазмонов $E_{\omega}$ в элементарной ячейке графеновой структуры при коэффициенте асимметрии $K=0.1$. го поля вблизи гибридного резонанса и к значительному увеличению выпрямленного тока (рис. 2,b).

При взаимодействии третьей „радиационной“ и третьей „нерадиационной“ плазмонных мод под широким затворным электродом (рис. 3, 4 и 5) в диапазоне частот $3.1-3.5$ ТГц (рис. 2) максимальный выпрямленный ток возникает при коэффициенте асимметрии 0.4 (рис. 2, $b$ ). Дело в том, что „нерадиационная“ мода (с большей частотой) в слабо асимметричной структуре имеет малый дипольный момент и поэтому слабо взаимодействует с другими модами и слабо возбуждается внешней ТГц волной (рис. 5). При увеличении асимметрии структуры дипольный момент „нерадиационной“ моды растет, в результате чего она может быть эффективно возбуждена полем падающей ТГц волны (рис. 3 и 4). В этом случае „нерадиационная“ мода становится оптически активной и происходит эффект антипересечения третьей „радиационной“ и иретьей „нерадиационной“ плазмонных мод (рис. 3 и 4). При сильной асимметрии структуры взаимодействие указанных мод уменьшается, в результате чего уменьшается асимметрия пространственного распределения поля и уменьшается выпрямленный ток (рис. 2, b). Наибольший эффект выпрямления достигается в случае, когда „радиационная“ и „нерадиационная“ моды эффективно взаимодействуют (рис. 4), приводя к образованию гибридной моды с резонансом типа Фано (рис. 2).

Чувствительность выпрямления ТГц излучения на единицу площади структуры графенового детектора $(300 \mathrm{~A} \cdot \mathrm{мm} / \mathrm{BT})$ превышает более чем на 2 порядка величины чувствительность детектора на основе массива полевых транзисторов $\mathrm{GaAs} / \mathrm{AlGaAs}$ [20] (для которого чувствительность на единицу площади составляет $\sim 1 \mathrm{~A} \cdot \mathrm{MM} / \mathrm{BT})$.

\section{4. Заключение}

Таким образом, в данной работе исследовано влияние геометрической асимметрии и асимметрии электрического поля плазмонов на выпрямление ТГц излучения за счет плазмонных эффектов в графеновой структуре с двойным решеточным затвором. Выяснено, что в структуре с участками электронной и дырочной проводимости в элементарной ячейке выпрямление ТГц излучения происходит главным образом за счет эффекта плазмонного электронно-дырочного храповика. При этом значительное увеличение чувствительности детектирования происходит при возбуждении гибридных плазмонных резонансов. Вычисленная чувствительность на единицу площади графеновой структуры более чем на 2 порядка величины превышает чувствительность плазмонного детектора на основе массива полевых транзисторов $\mathrm{GaAs} / \mathrm{AlGaAs}$.

Работа выполнена при поддержке грантов РФФИ (№ 15-02-02989 и 17-52-53063) и гранта президента РФ для молодых ученых (МК-5447.2016.2). 


\section{Список литературы}

[1] M. Dyakonov, M. Shur. IEEE Trans. Electron Dev., 43, 380 (1996).

[2] A.V. Muravjov, D.B. Veksler, V.V. Popov, O.V. Polischuk, N. Pala, X. Hu, R. Gaska, H. Saxena, R.E. Peale, M.S. Shur. Appl. Phys. Lett., 96, 042105 (2010).

[3] S. Boubanga-Tombet, Y. Tanimoto, A. Satou, T. Suemitsu, Y. Wang, H. Minamide, H. Ito, D.V. Fateev, V.V. Popov, T. Otsuji. Appl. Phys. Lett., 104, 262104 (2014).

[4] P. Olbrich, J. Kamann, M. Konig, J. Munzert, L. Tutsch, J. Eroms, D. Weiss, Ming-Hao Liu, L.E. Golub, E.L. Ivchenko, V.V. Popov, D.V. Fateev, K.V. Mashinsky, F. Fromm, Th. Seyller, S.D. Ganichev. Phys. Rev. B, 93, 075422 (2016).

[5] M. Sprinkle, D. Siegel, Y. Hu, J. Hicks, A. Tejeda, A. TalebIbrahimi, P. Le Fevre, F. Bertran, S. Vizzini, H. Enriquez, S. Chiang, P. Soukiassian, C. Berger, W.A. de Heer, A. Lanzara, E.H. Conrad. Phys. Rev. Lett., 103, 226803 (2009).

[6] V. Ryzhii, T. Otsuji, M. Ryzhii, M.S. Shur. J. Phys. D: Appl. Phys., 45, 302001 (2012).

[7] V. Ryzhii, T. Otsuji, M. Ryzhii, V. Mitin, M.S. Shur. J. Appl. Phys., 118, 204501 (2015).

[8] X. Cai, A.B. Sushkov, M.M. Jadidi, L.O. Nyakiti, R.L. MyersWard, D.K. Gaskill, T.E. Murphy, M.S. Fuhrer, H.D. Drew. Nano Lett., 15, 4295 (2015).

[9] A. Tomadin, A. Tredicucci, V. Pellegrini, M.S. Vitiello, M. Polini. Appl. Phys. Lett., 103, 211120 (2013).

[10] D.V. Fateev, K.V. Mashinsky, V.V. Popov. Appl. Phys. Lett., 110, 061106 (2017).

[11] V.V. Popov. J. Infrared Millim. Terahertz Waves, 32, 1178 (2011).

[12] V.V. Popov. Appl. Phys. Lett., 102, 253504 (2013).

[13] T. Watanabe, S.A. Boubanga-Tombet, Y. Tanimoto, D. Fateev, V. Popov, D. Coquillat, W. Knap, Y.M. Meziani, Y. Wang, H. Minamide, H. Ito, T. Otsuji. IEEE Sensors J., 13, 89 (2013).

[14] L. Vicarelli, M.S. Vitiello, D. Coquillat, A. Lombardo, A.C. Ferrari, W. Knap, M. Polin, V. Pellegrini, A. Tredicucci. Nature Materials, 11, 865 (2012).

[15] F. Bianco, D. Perenzoni, D. Convertino, S.L.D. Bonis, D. Spirito, M. Perenzoni, C. Coletti, M.S. Vitiello, A. Tredicucci. Appl. Phys. Lett., 107, 131104 (2015).

[16] S. Rudin. Int. J. High Speed Electron. Syst., 20, 567 (2011).

[17] G.R. Aizin, D.V. Fateev, G.M. Tsymbalov, V.V. Popov. Appl. Phys. Lett., 91, 163507 (2007).

[18] Д.В. Фатеев, В.В. Попов, M.S. Shur, ФТП, 44, 1455 (2010).

[19] V.V. Popov, D.V. Fateev, E.L. Ivchenko, S.D. Ganichev. Phys. Rev. B, 91, 235436 (2015).

[20] V.V. Popov, D.M. Ermolaev, K.V. Maremyanin, N.A. Maleev, V.E. Zemlyakov, V.I. Gavrilenko, S.Yu. Shapoval. Appl. Phys. Lett., 98, 153504 (2011).

Редактор Л.В. Шаронова

\section{Giant effect of terahertz radiation rectification in periodic graphene plasmonic structures}

D.V. Fateev ${ }^{1}$, K.V. Mashinsky ${ }^{1,2}$, Hua Qin $^{3}$, Jiandong Sun ${ }^{3}$, V.V. Popov ${ }^{1,2,4}$

\author{
${ }^{1}$ Kotelnikov Institute of Radio Engineering \\ and Electronics (Saratov Branch), \\ Russian Academy of Sciences, \\ 410019 Saratov, Russia \\ ${ }^{2}$ Saratov State University, \\ 410012 Saratov, Russia \\ ${ }^{3}$ Key Laboratory of Nanodevices and Applications, \\ Suzhou Institute of Nano-tech and Nano-bionics \\ (SINANO), Chinese Academy of Sciences, \\ 398 Ruoshui Road, 215123 Suzhou, P. R. China \\ ${ }^{4}$ Saratov Scientific Center, \\ Russian Academy of Sciences, \\ 410028 Saratov, Russia
}

Abstract The problem of rectification of terahertz radiation due to plasmonic nonlinearities in a periodic graphene structure with dual-grating gate and asymmetrical unit cell is solved theoretically. The influence of the unit cell asymmetry on rectification of terahertz radiation near high-order plasmon resonances for electron and hole graphene conductivity in the unit cell is studied. The current responsivity of the rectification of terahertz radiation due to both plasmonic effects of the charge carrier drag and the electron-hole ratchet is calculated. 